\title{
Extraction and Analysis of Alteration Information from Remote Sensing Imagery in Maoping lead-zinc Deposit, Yunnan province,China
}

\author{
Wei Ma ${ }^{1,2, a}$, Xingping Wen ${ }^{1,2, b^{*}}$, Zhenjie Shi ${ }^{1,2}$, Lijuan Zhang ${ }^{1,2}$, Jun Wang ${ }^{1,2}$, \\ Guanghui $\mathrm{Wu}^{3}$
}

${ }^{1}$ Faculty of Land Resource Engineering, Kunming University of Science and Technology, Kunming, Yunnan, 650093, China

${ }^{2}$ Mineral Resources Prediction and Evaluation Engineering Laboratory of Yunnan Province, Kunming, Yunnan, 650093, China

${ }^{3}$ Northwest University, State Key Laboratory of Continental Dynamics, Xi'an, 710069,China

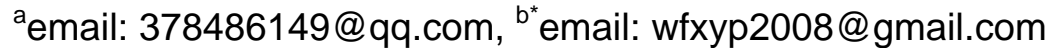

Keywords: Remote sensing; Alteration anomalies; principal component analysis;

\begin{abstract}
The paper based on the analysis of the regional geological background of Maoping lead-zinc deposit and extract alternation information in the area through ETM+ remote sensing image. It used principal component analysis to extract the alternative anomalies. We can research on the alternation anomalies and regional structure, the results shows that:1)there are only a small amount of alteration anomalies along with NE. 2) alteration anomalies distributed on NW and it has a certain scale. 3) mining alteration anomalies is focus on Linjiaping and Huamiaozhai, these can be identified as prospecting favorable position.
\end{abstract}

\section{Introduction}

Since the advent of remote sensing technology, Many kinds of remote sensing data contains the feature of spectral information, spatial information.Using this information will be conducive to the object information extraction. Alteration information is an important sign of prospecting.Looking for alteration and mineralization information plays an important role in geological prospecting.studying and learning feature is particularly important for finding large deposits or large ore concentration area.Thus, extraction information from remote sensing images can narrow the scope and improve the accuracy of prospecting.

\section{General Conditions of Study Area}

Maoping lead-zinc mine area is located in the middle of Wuxing-YiLiang and Maoping-Weixin which seated in Huize the northeast of Yunnan. The region northeast-southwest up 26km, its widest point is $10 \mathrm{~km}$. Luoze River through it, the mine is divided in two parts those. East is Maoping mine area, west is Hongjianshan-Chenjiawu foundation.

The land of mine exposed the upper and middle Devonian, the middle and lower Carboniferous, the middle and lower Permian.Along with NW Ziyun - Yadu fracture is the regional backbone structure,and Zhaotong-Qujing approaching north to south blind fault control the development of regional geological structure and the formation of the lead-zinc deposit, the secondary structure is the developed NE wrinkle and fracture, control lead-zinc deposits in metallogenic belt and the main distribution northeast Yunnan.

$\mathrm{NE}$ trending structures is developed in the mine, those is the Maomao mountain overturned anticline, Maoping fracture, Fangmaba fracture, Luoze river fracture. Another is NW faults, the representative is Longjie fracture[1]. 


\section{Survey of the study area}

Surrounding all types of alteration rocks, but the intensity is weak, one of the most significant is the dolomitization and pyrite phenomenon which is relate to the development and distribution on Maoping mine area. So alteration studies will commence around existing information that Hydrocarbon alteration (dolomitization) and iron stained alteration (pyrite); alteration information extraction using principal component analysis[2][3].

\section{Extraction alteration information of Hydrocarbon}

Hydroxy[4] (mudding) alteration information mainly concentrated in the far infrared band, because the interaction between the iron-based compound and visible is the most prominent, to distinguish and identify carbonate and Hydrocarbon radicals and iron minerals effectively, select the ETM+1,4,5,7band making component transform; there are a lot of confounding factors in the area, in order to eliminate false portent as much as possible, apply the comprehensive mask in the component transformation process. That is, the unmasked data information should be transformed effectively and extract the component PC1-PC4.

After sensing image of the mask be treated, choosing Band1,4,5,7 analysis the component, statistical results are as follows:

\begin{tabular}{ccccc} 
& Table1 & ETM+ 1、4、5、7 & \\
\hline Eigenvectors & Band 1 & Band 4 & Band 5 & Band 7 \\
\hline PC1 & -0.051511 & -0.060287 & -0.144382 & -0.986340 \\
PC2 & 0.243652 & 0.851179 & 0.446326 & -0.130084 \\
PC3 & 0.177148 & 0.426193 & -0.882142 & 0.037529 \\
PC4 & 0.952155 & -0.300367 & 0.042098 & -0.037529 \\
\hline
\end{tabular}

From Table1, B7 feature vector is positive in PC4 , B7 feature vector is negative, on the PC4 image hydroxy abnormal grading extraction[5]. The statistical analysis shows that, PC4 standard deviation of 7.418560 on gray scale images, perform grading standard deviation based on a multiple of:

The anomalies: $0+3 * 7.418560=22.25568$

\section{Extraction alteration information of iron stained}

After a comprehensive mask, according to the differences in the different bands of iron alteration minerals reflection, remote sensing information[6] about the iron stained can be extracted. Thus ETM+1,3,4,5 are used to component analysis. Statistical results are as follows:

Table2 ETM+ 1、3、4、5

\begin{tabular}{ccccc}
\hline Eigenvectors & \multicolumn{1}{c}{ Band 1 } & \multicolumn{1}{c}{ Band 3 } & Band 4 & \multicolumn{1}{c}{ Band 5 } \\
\hline PC1 & 0.284150 & 0.422413 & 0.723305 & 0.466536 \\
PC2 & -0.277356 & -0.670679 & 0.648579 & -0.229365 \\
PC3 & -0.524504 & -0.228314 & -0.177173 & 0.800161 \\
PC4 & 0.753146 & -0.565358 & -0.157429 & 0.297250 \\
\hline
\end{tabular}

From Table 2, B5 feature vector is positive on PC4, B5feature vector is negative, about the iron stained alteration information The statistical analysis shows that, PC4 standard deviation of 3.080582 on gray scale images, perform grading standard deviation based on multiples:

The anomalies: $0+3 * 3.080582=9.241746$ 


\section{Results and Discussion}

Extraction and analysis the alteration information like hydroxyl, iron stained at Maoping, Zhaotong. Research on the intensity of the alteration information, the development of the construction, the distribution of the construction, etc. The results analysis about the alteration information in the area are follows:

1) NE trending fracture developed in the area is less, and the size is small. only a small amount of alteration anomalies in Guanying mountain along with NE.

2) Alteration anomalies distributed along NW orientation, and the directional breaking output and has a certain scale. About this, Jiangjiawang in the southwest, Linjiaping in the southwest both exist alteration.

3) According to the landform, structure, alteration and other information, extraction alteration anomalies and research on regional structure, comprehensive analysis, mining abnormal alteration information is focus on Linjiaping and Huamiaozhai, these can be identified as prospecting favorable positions.

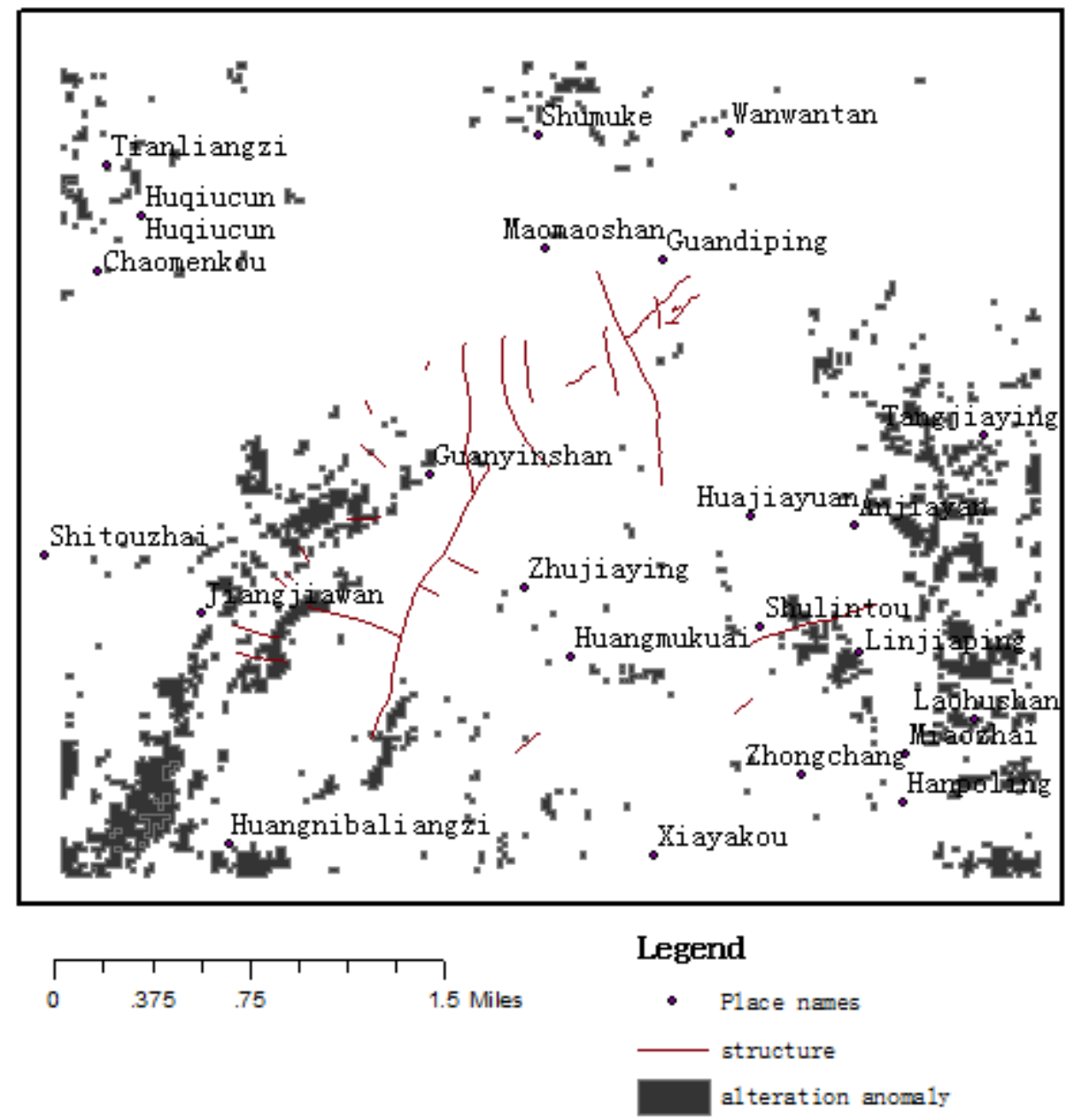

\section{Acknowledgement}

This study was jointly supported by Natural Science Foundation of China (Grant No. 41101343), the remote sensing geochemistry disciplinary innovation team, Kunming University of Science and Technology, Kunming, China, 2013, and the innovation team of ore-forming dynamics and prediction of concealed deposits, Kunming University of Science and Technology, Kunming, China, 2008. 


\section{References}

[1] Hechang Liu, Wenda Lin. Law of $\mathrm{Pb}$ and $\mathrm{Zn}$ in northeast Yunnan Research [M]. Yunnan: YunnanUniversity Publisher, 1999.

[2] Crosta A P, Moore J M. Enhancement of Landsat Thematic Mapper Imagery for Residual Soil Mapping in SW mINAS cERAIS [c]// Proceedings of the $7^{\text {th }}$ (ERIM) Thematic conference: Remote Sensing for Exploration Geology.Calgar,1989:225-255.

[3] Loughlin W p.Principal CompentAnalysis for Alteration Mapping[J]. Photogrammetric Engineering and Remote Sensing, 1991,57(9): 163-1169

[4] Tommasoe I D, Rubinstein n. Hydrothermal Alteration Mapping Using ASTER Data in the Infiernillo Porphyry Deposit, Argentina[J]. Ore Geology Reviews,2007, 32(1-2); 275-290.

[5] Vapnik Vn. Statical Learning Theory [M]. New York:Wiley, 1998:3-55.

[6] Yunfeng Zhang, Lingjun Li,Rong Zhang, Semiquantitative Application of Romote sensing Structure Explanation to ore Prediction in northeast Yunnan area.[J] Mineral Resources and Geology.Vol21, No.23,2007 